\title{
Planificación de recursos hídricos en zonas de secano usando un modelo de optimización no lineal
}

\section{Planning water resources in dry lands using a non-linear optimization model}

Fecha de entrega: 22 de junio 2011

Fecha de aceptación: 6 de octubre 2011

\author{
Abelardo Villavicencio ${ }^{1}$, José Luis Arumín ${ }^{2}$ y Eduardo Holzapfel ${ }^{2}$ \\ ${ }^{1}$ Instituto de Investigaciones Agropecuarias INIA Rayentué, Avda. Salamanca s/n, km 105 ruta 5 sur, Los Choapinos, Casilla 13, \\ Rengo, Chile, avillavi@inia.cl \\ ${ }^{2}$ Facultad de Ingeniería Agrícola, Universidad de Concepción, Casilla 537, Chillán, Chile, jarumi@udec.cl, eholzapf@udec.cl
}

Se desarrolló una metodología de trabajo que permite identificar y planificar el uso de los recursos hídricos en zonas de secano, haciendo uso de una base de datos con información de un conjunto de cuencas identificadas con el software ARCVIEW 3.2. Se obtuvo información meteorológica de la zona de estudio que fue utilizada en un balance hídrico para cuantificar la cantidad potencial de agua a acumular en cada sitio identificado. Posteriormente se usó un modelo de optimización no lineal que determina un patrón de cultivo que maximiza el beneficio neto dado un conjunto de recursos y restricciones físicas, medioambientales y económicas. A través de un análisis de sensibilidad se encontró que las variables que más influyen sobre el beneficio neto son el precio de venta de los productos agrícolas, la disponibilidad de agua, la disponibilidad de mano de obra y la eficiencia del método de riego. También se determinó que las cuencas de tipo pequeño son más sensibles a los cambios en estas variables. Una estrategia adecuada de intervención en el secano es introducir especies que se adapten a esta condición y utilizar el agua acumulada para dar riegos suplementarios en épocas de mayor demanda hídrica. No se pretende transformar toda el área en una zona de riego, sino que desde sus limitantes y potencialidades planificar el uso racional del agua factible de acumular.

Palabras clave: recursos hídricos, funciones de producción, modelos de optimización, planificación, secano, sistema de información geográfica
A methodology was developed for identifying and planning the water resources use in dry lands. A data base was constructed with information of small basins which were identified using the ARCVIEW 3.2 software. Meteorological information was used to estimate run-off and to calculate the potential water volume that could be accumulated in each identified site. Subsequently, a non linear optimization model was used to determine the crop pattern that maximizes the net benefit according to the physical, environmental and economic resources and constraints. The sensibility analysis of the model indicated that the most important variables were product price, water availability, labor hand availability and irrigation method efficiency. It was also determined that small basins are more sensible than medium or big basins. An appropriate intervention strategy for dry lands is to introduce adapted species to these conditions and to use captured water for supplementary irrigation in the few months with high water demand. The idea is not to transform the whole area in an irrigation zone, but from its limitations and potentialities to plan a rational use of the water that can be captured in winter.

Keywords: water resources, production functions, optimization models, planning, dry lands, geographic information systems

limitante. En general estas zonas presentan altos niveles de pobreza por lo que se busca incorporar alternativas de mayor rentabilidad que contribuyan a la diversificación de los sistemas productivos, centrados actualmente en cultivos tradicionales como trigo y garbanzos que tienen
Las condiciones de sequía, fenómeno cada vez más recurrente en Chile, debe hacernos reflexionar sobre como abordar de forma más eficiente la producción agrícola en zonas donde la restricción de agua es una severa 
baja rentabilidad. Es necesario enfrentar técnicamente el problema de como obtener una mejor producción agrícola en zonas de restricción hídrica. Para esto se deben utilizar estrategias como, incorporar sistemas de riego tecnificado y capturar aguas producto de la escorrentía superficial del invierno para ser utilizadas en épocas de déficit hídrico (Billib et al., 2009).

La tecnología disponible permite ocupar poderosas herramientas como los sistemas de información geográfica y modelos de optimización, capaces de manejar gran cantidad de información y de generar diferentes escenarios a partir de los cuales se pueden establecer políticas de apoyo o fomento, tal que ayuden a mitigar el problema de escasez de agua para fines agrícolas o de consumo humano.

El objetivo de este trabajo es desarrollar una metodología que permita identificar y planificar el uso de los recursos hídricos en condiciones de secano. Esta metodología se desarrolló en dos comunas de la Región de O’Higgins, llamadas Navidad y Pumanque (ver ubicación en Figura 1). La metodología desarrollada tiene dos componentes: 1) la confección de una base de datos que contiene información referenciada espacialmente de un conjunto de sectores donde se pueden construir obras de almacenamiento (embalses); y 2) desarrollar un modelo de optimización para determinar el patrón óptimo de cultivos que, en los sitios identificados, maximice las utilidades.

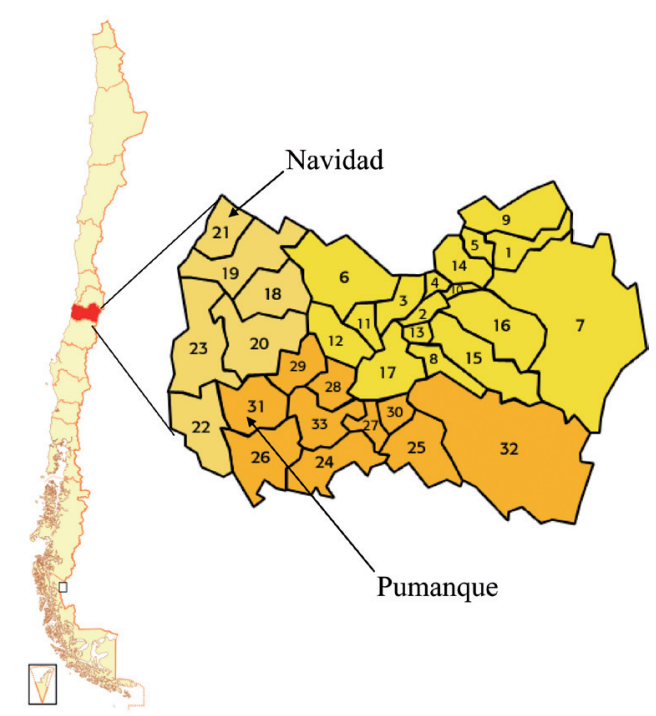

Figura 1: Ubicación de las dos comunas estudiadas de la región de O’Higgins

\section{Área de estudio}

El área de estudio comprendió la comuna de Navidad en la zona de secano costero y la comuna de Pumanque en el secano interior. El clima en estas zonas se caracteriza por una estación seca y calurosa entre los meses de septiembre y marzo que corresponden a la primavera y verano del Hemisferio Sur. En el sector costero se aprecia la influencia oceánica que determina menores variaciones térmicas diarias e intermensuales. En cuanto a las precipitaciones, se caracterizan por su variabilidad y se concentran en invierno, entre abril y julio, alcanzando niveles de $660 \mathrm{~mm}$ al año aproximadamente en la comuna de Navidad y $518 \mathrm{~mm}$ en Pumanque, como promedios de los últimos 30 años.

La hidrología de la zona de estudio es similar a la descrita por Uribe et al. (2003). Los suelos que forman las cuencas son predominantemente graníticos, con una baja infiltración. Por esta razón, durante los eventos de precipitación invernal, se producen fuertes crecidas y en verano prácticamente no existe flujo, lo cual justifica el uso de estructuras de acumulación.

\section{Metodología}

\section{Identificación de posibles localizaciones de embalses}

La información cartográfica básica utilizada para este estudio se obtuvo del Instituto Geográfico Militar de Chile y la información meteorológica de la Dirección de Aguas, dependiente del Ministerio de Obras Públicas. Utilizando el software ARC VIEW $3.2 \AA^{\circledR}$, se confeccionó un mapa base digital de la zona que contiene la información relevante para el estudio. Utilizando el plano base, se seleccionaron ubicaciones de posibles obras de almacenamiento de acuerdo al siguiente esquema:

1. En cada comuna se identificó un conjunto de pequeñas cuencas cuyas aguas drenaran hacia sectores donde existiera población y que fuera posible realizar una actividad agrícola.

2. En cada cuenca, utilizando la información de curvas de nivel, se identificaron puntos donde se producen estrechamientos del cauce de los esteros que pueden ser aprovechados para la construcción de muros de embalses.

3. En zonas de alta pendiente el volumen factible de ser acumulado es muy limitado. Por esta razón, para este estudio, se descartaron como posibles ubicaciones de 
embalses todas aquellas áreas que presentaban una pendiente del cauce superior al 10\%.

Para estimar la escorrentía de cada cuenca se realizó un balance hídrico mensual(Uribe etal., 2003). La información básica se obtuvo del mapa base generado inicialmente y de datos meteorológicos recogidos en estaciones cercanas a la zona de estudio.

\section{Modelo de optimización}

Para determinar el uso más eficiente del agua, se utilizó un modelo de optimización no lineal que usa funciones aguaproducción para diversos cultivos, costos de producción y costo de construcción de embalses y determina el patrón de cultivos que optimice el uso del agua. Dicho modelo de optimización fue ejecutado mediante el software GAMSMINOS 2.25 (Brooke et al., 1988). Las funciones agua producción para los cultivos de cerezo, frambueso, alfalfa, trigo y maíz se obtuvieron de Carvallo et al. (1998). Mientras que para cebolla y almendro, los datos fueron extraídos de Al-Jamal et al. (1999) y Torrecillas et al. (1989) respectivamente.

Para facilitar la parametrización y la comparación de la información, los rendimientos y la evaporación de bandeja fueron expresados en forma relativa respecto a un máximo potencial establecido para la zona en base a los antecedentes históricos de producción. Las funciones de producción consideradas en el modelo tienen la siguiente expresión:

$$
\begin{aligned}
& Y_{R E L}=A C+B C\left(E B_{R E L}\right)^{a}-C C\left(E B_{R E L}\right)^{b} \\
& Y=\left\{A C+B C\left(E B / E B_{\text {max }}\right)^{a}-C C\left(E B / E B_{\text {max }}\right)^{b}\right\} Y_{\text {max }}
\end{aligned}
$$

donde $Y$ es el rendimiento del cultivo en $\mathrm{kg} / \mathrm{ha}$ (o ton/ha o qqm/ha), $Y_{\text {máx }}$ corresponde a los rendimientos máximos en $\mathrm{kg} / \mathrm{ha}, Y_{R E L}=Y / Y_{\text {max }}$ es el rendimiento relativo, $E B$ es la evaporación de bandeja en $\mathrm{mm}$ de agua, $E B_{\text {máx }}$ es la evaporación de bandeja máxima o potencial del área en mm de agua, $E B_{R E L}=E B / E B_{\text {máx }}$ es la evaporación de bandeja relativa y $A C, B C, C C, a$ y $b$ son coeficientes determinados experimentalmente. La función objetivo es representada por la siguiente expresión:
$\operatorname{Max} U=\sum_{i=1}^{n}\left(P_{i} A_{i} Y_{i}-C_{i} A_{i}\right)$

donde $\mathrm{U}$ es la utilidad en US\$, $P_{i}$ es el precio del producto $i$ en US\$/unidad de producción, $A_{i}$ es el área sembrada del cultivo $i$ en ha, $Y_{i}$ es la producción del cultivo $i$ en unidades de producción/ha, $C_{i}$ es el costo de los insumos utilizados en el cultivo $i$ en US\$/ha, $i$ es el número entero correspondiente al tipo de cultivo $(1,2, \ldots ., n)$ y $n$ es el número total de cultivos considerados. Si se sustituye la expresión (2) en (3) se tiene:

$$
\begin{aligned}
& M a x U=\sum_{i=1}^{n} P_{i} A_{i}\left(A C_{i}+B C_{i}\left(E B / E B_{\max }\right)^{a} .\right. \\
& \left.-C C_{i}\left(E B / E B_{\max }\right)^{b}\right) Y_{\max }-\sum_{i=1}^{n} C_{i} A_{i}
\end{aligned}
$$

Los costos de producción se dividen en 4 tipos:

i. Costo del agua embalsada $\mathrm{CW}$, en US $\$ / \mathrm{m}^{3}$, expresada mediante la siguiente función de costo (CIREN CORFO, 1996)

$$
C W=124.73 W_{i}^{0.4866}
$$

donde $W_{i}$ es el volumen de agua a embalsar.

ii. Costo de implementación del método de riego empleado CR, en US\$/ha

iii. Costo de mano de obra CMO, en US\$/JH

iv. Otros costos: maquinaria, fertilizantes, pesticidas y semillas; OC, en US\$/ha

Incluyendo estas expresiones en (4) con los costos arriba mencionados se obtiene:

$$
\begin{aligned}
& \operatorname{Max} U=\sum_{i=1}^{n} P_{i} A_{i}\left(A C_{i}+B C_{i}\left(E B / E B_{\text {max }}\right)^{a}-C C_{i}\left(E B / E B_{\text {max }}\right)^{b}\right) Y_{\text {max }} \\
& -\sum_{i=1}^{n}\left(C W_{i} A_{i} W_{i}-A_{i} C R_{i}-A_{i} C M O_{i} N M O_{i}-A_{i} O C_{i}\right)
\end{aligned}
$$

donde $N M O_{i}$ es la mano de obra por hectárea necesaria para el cultivo $i, W_{\mathrm{i}}$ es la necesidad bruta de agua para el cultivo $i$ en $\mathrm{m}^{3} / \mathrm{ha}, C R_{i}$ son costos de riego anuales del cultivo $i$ asociado al método de riego $j$, y $O C_{i}$ otros costos asociados al cultivo $i$. Los costos de riego $C R_{i}$ asociados a los métodos de riego por goteo y aspersión corresponden a los costos totales anuales por hectárea de los equipos, 
considerando una vida útil de 20 años. Las necesidades brutas de agua de riego $W_{i}$, para cada cultivo se obtuvieron mediante la siguiente relación:

$$
W_{i}=10 \frac{E B_{i}}{E A_{i}}
$$

donde $E B_{\mathrm{i}}$ son requerimientos de agua para el cultivo $i$ en mm y $E A_{i}$ es la eficiencia de aplicación de agua del método de riego usado para regar el cultivo $i$ en porcentaje $\%$.

Finalmente, la función objetivo del modelo queda expresada como,

$$
\begin{aligned}
& \operatorname{Max} U=\sum_{i=1}^{n} P_{i} A_{i}\left\{A C_{i}+B C_{i}\left(E B / E B_{\max }\right)^{a}-C C_{i}\left(E B / E B_{\max }\right)^{b}\right\} Y_{\max i}- \\
& 10 \sum_{i=1}^{n}\left(C W_{i} \frac{E B}{E A_{i}} A_{i} W_{i}\right)-\sum_{i=1}^{n}\left(A_{i} C R_{i}-A_{i} C M O_{i} N M O_{i}-A_{i} O C_{i}\right)
\end{aligned}
$$

Además se consideraron restricciones físicas, de manejo, económicas, diversificación productiva y disponibilidad de recursos. Estas fueron:

\section{a) Disponibilidad de agua}

La restricción física de la disponibilidad de agua debe considerar que la suma de todos los requerimientos brutos de agua de los cultivos $i$, no puede ser mayor a la disponibilidad total de agua embalsada $W_{t}\left(m^{3}\right)$. Los requerimientos brutos de agua se calcularon a partir de la relación entre las necesidades netas de agua expresadas en forma de evaporación de bandeja $E B$ y la eficiencia del método de riego utilizado $E A$. Por lo tanto la restricción es:

$$
\sum_{i=1}^{n} \frac{E B_{i}}{E A_{i}} A_{i} 10 \leq W_{i}
$$

\section{b) Disponibilidad de suelos}

La restricción de suelo se expresó de la siguiente forma:

$$
\sum_{i=1}^{n} A_{i} \leq S_{t}
$$

donde $S_{t}$ es la superficie disponible en ha y $A_{i}$ es la superficie destinada al cultivo $i$

\section{c) Disponibilidad de mano de obra}

La restricción de este recurso depende de la fuerza de trabajo disponible en la zona. Su expresión es:

$$
\sum_{i=1}^{n} N M O_{i} A_{i} \leq D T M O
$$

donde $D T M O$ es la disponibilidad total anual de mano de obra $J H$.

d) Criterios de manejo, mercado y diversificación productiva

Desde el punto de vista práctico es necesario considerar ciertos criterios de manejo agrícola, de mercado y de diversificación productiva para restringir las áreas máximas o mínimas de cultivo. Esto se debe a situaciones de comercialización, mercado, rotaciones u otras limitantes agronómicas. Estas restricciones se expresan como:

$$
\min S_{i} \leq \sum_{i=1}^{m} A_{i} \leq \max S_{i}
$$

donde $\min S_{i}$ es la mínima área a cultivar con el cultivo $i$ y $\max _{i}$ es la máxima área a cultivar con el cultivo $i$.

\section{Datos básicos de entrada al modelo}

Los datos de entrada del modelo de optimización, costos y parámetros técnicos se presentan en la Tabla 1. Los rendimientos potenciales se consideran bajo niveles óptimos de riego y manejo agronómico. Las especies frutales son regadas por goteo y el resto por aspersión. El costo de mano de obra considerado es de $7 \mathrm{US} \$ / \mathrm{JH}$, que corresponde al salario promedio que se paga en las actividades agrícolas chilenas.

Tabla 1: Datos de entrada del modelo de optimización, costos y parámetros técnicos

\begin{tabular}{|l|c|c|c|c|c|}
\hline Cultivo & $\begin{array}{c}\text { Req. } \\
\text { mano } \\
\text { obra JH/ } \\
\text { ha }\end{array}$ & $\begin{array}{c}\text { Costo } \\
\text { riego } \\
\text { US\$/ha }\end{array}$ & $\begin{array}{c}\text { Otros } \\
\text { costos } \\
\text { US\$/ha }\end{array}$ & $\begin{array}{c}\text { Precio } \\
\text { US\$/ } \\
\mathrm{kg}\end{array}$ & $\begin{array}{c}\text { Rend. } \\
\text { máx. } \\
\mathrm{kg} / \mathrm{ha}\end{array}$ \\
\hline Cerezo & 120 & 287 & 583,7 & 1,14 & 10000 \\
Almendro & 51 & 287 & 284,2 & 3,5 & 5000 \\
Frambueso & 460 & 287 & 385,4 & 1.37 & 8000 \\
Alfalfa & 12,5 & 215 & 347,3 & 0,08 & 16000 \\
Cebolla & 116 & 215 & 657,9 & 0,19 & 50000 \\
Trigo & 7,1 & 215 & 477,0 & 0,18 & 6000 \\
Maíz & 14,5 & 215 & 618,7 & 0,19 & 12000 \\
& & & & & \\
\hline
\end{tabular}




\section{Funciones agua - producción}

Las funciones agua - producción usadas en el modelo se muestran en la Tabla 2 y están basadas en la evaporación de bandeja relativa $E_{\text {REL }}$ y rendimiento relativo $\mathrm{Y}_{\mathrm{REL}}$.

Tabla 2: Funciones agua - producción usadas en el modelo, basadas en la evaporación de bandeja relativa $\mathrm{EB}_{\mathrm{REL}} \mathrm{y}$ rendimiento relativo $\mathrm{Y}_{\mathrm{REL}}$

\begin{tabular}{|l|l|}
\hline Cultivo & Función \\
\hline Cerezo $^{\mathrm{a}}$ & $\mathrm{Y}_{\mathrm{REL}}=-0,0286+3,1661 \mathrm{~EB}_{\mathrm{REL}}-2,4364\left(\mathrm{~EB}_{\mathrm{REL}}\right)^{2}$ \\
Almendro $^{\mathrm{b}}$ & $\mathrm{Y}_{\mathrm{REL}}=3,3351 \mathrm{~EB}_{\mathrm{REL}}-2,8452\left(\mathrm{~EB}_{\mathrm{REL}}\right)^{2}$ \\
Frambueso $^{\mathrm{a}}$ & $\mathrm{Y}_{\mathrm{REL}}=-0,7473+7,6951 \mathrm{~EB}_{\mathrm{REL}}-8,7241\left(\mathrm{~EB}_{\mathrm{REL}}\right)^{2}$ \\
Alfalfa $^{\mathrm{a}}$ & $\mathrm{Y}_{\mathrm{REL}}=-0,0114+2,5343 \mathrm{~EB}_{\mathrm{REL}}-1,5875\left(\mathrm{~EB}_{\mathrm{REL}}\right)^{2}$ \\
Cebolla $^{\mathrm{c}}$ & $\mathrm{Y}_{\mathrm{REL}}=1,4993 \mathrm{~EB}_{\mathrm{REL}}-0,4963\left(\mathrm{~EB}_{\mathrm{REL}}\right)^{2}$ \\
Trigo $^{\mathrm{a}}$ & $\mathrm{Y}_{\mathrm{REL}}=0,1+1,9 \mathrm{~EB}_{\mathrm{REL}}-\left(\mathrm{EB}_{\mathrm{REL}}\right)^{2}$ \\
Maíz $^{\mathrm{a}}$ & $\mathrm{Y}_{\mathrm{REL}}=-0,35+2,21 \mathrm{~EB}$ \\
$\mathrm{REL}$ & $-0,91\left(\mathrm{~EB}_{\mathrm{REL}}\right)^{2}$ \\
\hline
\end{tabular}

${ }^{\mathrm{a} C}$ Carvallo et al. (1998), ${ }^{\mathrm{b}}$ Torrecillas et al. (1989), ${ }^{\mathrm{C}} \mathrm{Al}-\mathrm{Jamal}$ et al. (1999)

\section{Resultados y discusión}

Se identificaron 19 puntos potenciales para la construcción de embalses en la comuna de Navidad y 20 en la comuna de Pumanque, los cuales son mostrados en las Figuras 2 y 3. Cada uno de los puntos define el cierre de una cuenca aportante cuyas características y rendimientos son mostradas en las Tablas 3 y 4 . El volumen anual de escorrentía se calculó para una probabilidad de excedencia de $85 \%$, que es el valor de referencia tradicional que se utiliza en Chile para determinar la superficie bajo riego.

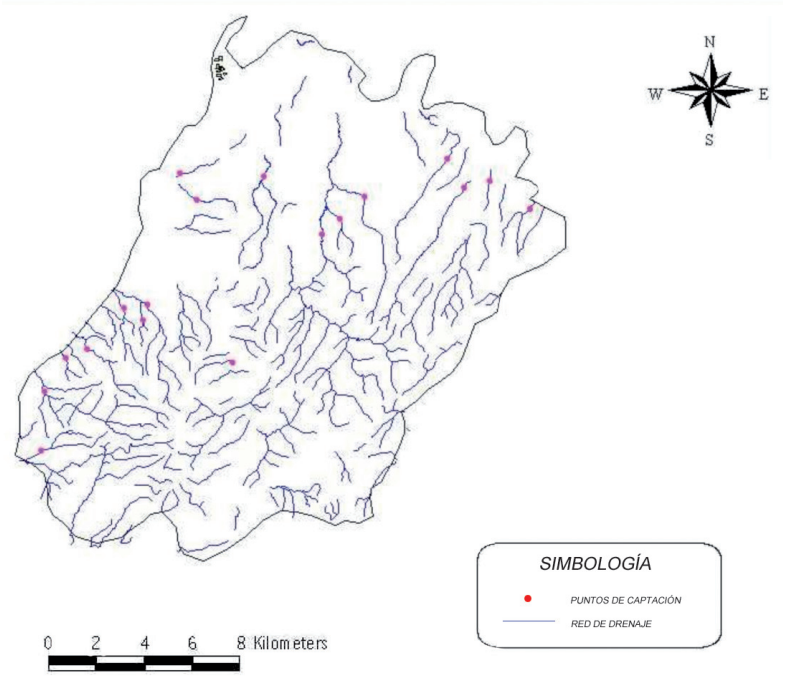

Figura 2: Puntos factibles para la construcción de acumuladores en la comuna de Navidad

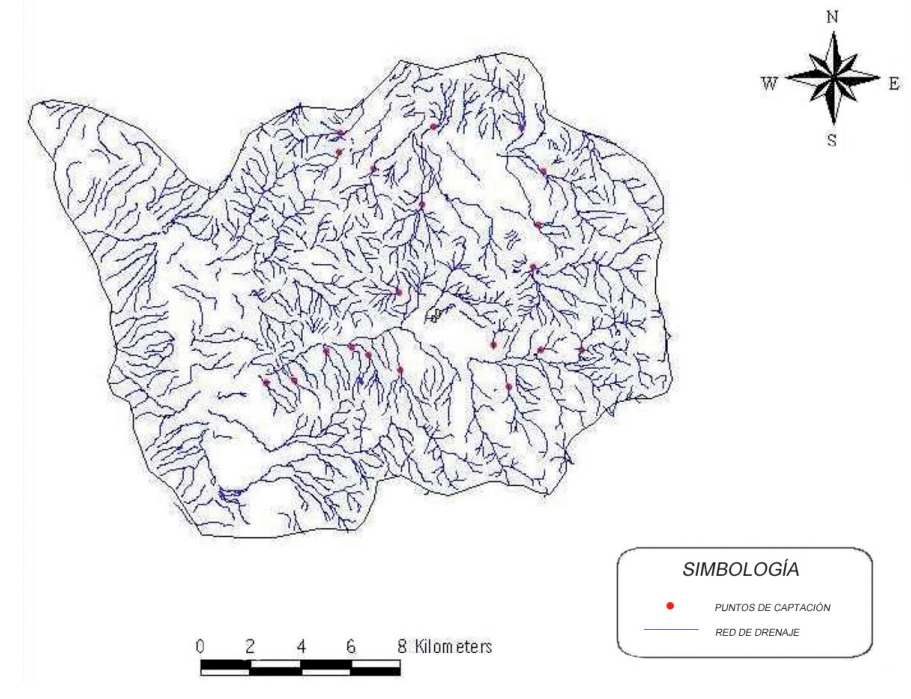

Figura 3: Puntos factibles para la construcción de acumuladores en la comuna de Pumanque

Tabla 3: Atributos de las cuencas identificadas para la comuna de Navidad

\begin{tabular}{|l|c|c|c|c|}
\hline Cuenca & $\begin{array}{c}\text { Perímetro } \\
\mathrm{km}\end{array}$ & $\begin{array}{c}\text { Area } \\
\mathrm{km}^{2}\end{array}$ & $\begin{array}{c}\text { Pendiente sector } \\
\mathrm{del} \mathrm{muro,} \mathrm{\%}\end{array}$ & $\begin{array}{c}\text { Escorrentía } \\
\mathrm{m}^{3} / \mathrm{año}\end{array}$ \\
\hline Centinela 1 & 6,3 & 2,8 & 3,4 & 28.661 \\
Centinela 2 & 11,3 & 4,6 & 2,8 & 47.597 \\
Chorrillos 1 & 7,5 & 2,2 & 2,7 & 22.660 \\
Chorrillos 2 & 12,6 & 4,8 & 0,9 & 49.976 \\
El Macho & 3,6 & 0,9 & 7,7 & 9.209 \\
El Mal Paso & 8,6 & 3,2 & 2,9 & 33.007 \\
Huehuinco & 6,2 & 1,9 & 7,5 & 20.073 \\
La Chépica & 15,3 & 9,3 & 5,3 & 96.228 \\
La Pulpería & 3,2 & 0,5 & 6,6 & 5.380 \\
La Vinilla & 14,7 & 6,5 & 1,4 & 67.256 \\
Los Queñes & 10,8 & 5,5 & 2,2 & 57.323 \\
Maitencillo & 4,6 & 1,4 & 5,1 & 13.969 \\
Matanza & 7,5 & 2,8 & 2,6 & 29.075 \\
Navidad & 31,0 & 31,3 & 1,0 & 323.864 \\
Pataguilla & 10,5 & 5,5 & 1,1 & 57.116 \\
Polcura & 3,3 & 0,5 & 8,0 & 5.484 \\
Pupuya & 21,7 & 23,3 & 1,3 & 241.087 \\
Rincón Polcura & 6,9 & 2,4 & 6,7 & 24.833 \\
San Rafael & 13,7 & 6,4 & 5,5 & 66.325 \\
\hline
\end{tabular}

Basándose en la superficie de las cuencas identificadas y su escorrentía media, en la comuna de Navidad las cuencas se agruparon en cuatro categorías: pequeña, mediana, medianagrande y grande; en la comuna de Pumanque se identificaron tres categorías: pequeña, mediana y grande (Tabla 5). La clasificación en diferentes categorías se hizo con la finalidad de facilitar el análisis y la aplicación del modelo de optimización cuyos resultados se indican en la Tabla 6. 
Tabla 4: Atributos de las cuencas identificadas para la comuna de Pumanque

\begin{tabular}{|l|c|c|c|c|}
\hline Cuenca & $\begin{array}{c}\text { Perímetro } \\
\mathrm{km}\end{array}$ & $\begin{array}{c}\text { Área } \\
\mathrm{km}^{2}\end{array}$ & $\begin{array}{c}\text { Pendiente sector } \\
\text { del muro, \% }\end{array}$ & $\begin{array}{c}\text { Escorrentía } \\
\mathrm{m}^{3} / \mathrm{año}\end{array}$ \\
\hline Alto del Peumo & 10,2 & 5,7 & 4,2 & 99.944 \\
Alto Las Vegas & 14,1 & 10,1 & 5,4 & 177.093 \\
Colhue & 12,8 & 8,1 & 2,1 & 142.025 \\
Com. M. Rodríguez 1 & 6 & 1,5 & 2,8 & 38.399 \\
Com. M. Rodríguez 2 & 6,1 & 2,2 & 2,1 & 26.301 \\
El Membrillo & 6,8 & 2,4 & 2,0 & 42.082 \\
La Esperanza & 14,8 & 10,6 & 2,0 & 185.860 \\
La Gloria & 7,1 & 3,3 & 5,6 & 57.862 \\
Loma de Itata & 7,2 & 3,5 & 4,3 & 61.369 \\
Loma El Clavo & 5 & 1,7 & 8,0 & 29.808 \\
Loma Jiménez & 4,8 & 1,2 & 7,9 & 21.041 \\
Los Pequenes & 5,4 & 1,9 & 2,2 & 33.315 \\
Orilla de los Silva & 17,5 & 10,5 & 0,8 & 184.107 \\
Palmilla de Reto & 12,2 & 8,0 & 1,3 & 140.272 \\
Peñablanca & 8,6 & 4,1 & 1,5 & 71.889 \\
Pta. Piedra & 4,9 & 1,4 & 4,4 & 24.548 \\
Punta Alta & 6,1 & 2,1 & 1,6 & 36.821 \\
Quetecura & 10,2 & 6,1 & 2,1 & 106.957 \\
Rincón La Mina & 9,1 & 4,5 & 5,7 & 40.328 \\
Sta. Esmeralda & 12,3 & 4,8 & 3,0 & 84.163 \\
\hline
\end{tabular}

Tabla 5: Clasificación de cuencas según superficie y comuna

\begin{tabular}{|c|c|c|l|}
\hline Comuna & $\begin{array}{c}\text { Rango de } \\
\text { superficie, } \mathrm{km}^{2}\end{array}$ & $\begin{array}{c}\mathrm{N}^{\mathrm{o}} \mathrm{de} \\
\text { cuencas }\end{array}$ & Clasificación \\
\hline \multirow{3}{*}{ Navidad } & $0,5-3,2$ & 10 & Pequeña \\
& $3,2-9,3$ & 7 & Mediana \\
& 23,3 & 1 & Mediana-Grande \\
& 31,3 & 1 & Grande \\
\hline \multirow{3}{*}{ Pumanque } & $1,2-3,5$ & 10 & Pequeña \\
& $3,5-8,1$ & 7 & Mediana \\
& $8,1-10,6$ & 3 & Grande \\
\hline
\end{tabular}

Tabla 6: Patrón de cultivo, por tipo de cuenca, resultado del modelo de optimización

\begin{tabular}{|l|c|c|c|c|c|c|c|c|}
\hline & & \multicolumn{5}{|c|}{ Comuna de Navidad } & \multicolumn{3}{c|}{ Comuna de Pumanque } \\
\hline & Unid. & Grande & Med-Gde & Med. & Pequeña & Grande & Med. & Med. \\
\hline Utilidad & USM\$ & 912,689 & 672,119 & 162,520 & 42,606 & 526,257 & 278,689 & 88,265 \\
\hline $\begin{array}{l}\text { Max. } \\
\text { superf. }\end{array}$ & ha & 61,6 & 45,8 & 12 & 3,6 & 39,5 & 20,4 & 7,1 \\
\hline $\begin{array}{l}\text { Agua } \\
\text { utilizada }\end{array}$ & $\mathrm{Mm}^{3}$ & 323,864 & 241,087 & 63,117 & 19,235 & 182,353 & 107,542 & 37,443 \\
\hline $\begin{array}{l}\text { Req. } \\
\text { mano } \\
\text { obra }\end{array}$ & $\mathrm{JH}$ & 3142 & 2339 & 612 & 186 & 2016 & 1043 & 363 \\
\hline Almendro & ha & 61,6 & 45,8 & 12 & 3,6 & 39,5 & 20,4 & 7,1 \\
\hline
\end{tabular}

Los resultados permitan apreciar que en todos los casos el patrón de cultivo recomendado está definido solo por el frutal almendro, lo que se explica por su alto precio de venta, menores costos de producción y baja demanda hídrica. Si bien es cierto este resultado genera el más alto valor de beneficio neto, deben considerarse razones agronómicas, de manejo, mercado y disponibilidad de recursos que hace aconsejable introducir algunos criterios de diversificación productiva que eviten la alta concentración de labores agrícolas en un corto período de tiempo y la sobreoferta de producto que haría disminuir su precio. Dado lo anterior, se establecieron límites máximos de superficie por especie, por su bajo precio y carácter extensivo de la producción los cultivos anuales se restringieron a un 5\% de la superficie total, por su alta demanda de mano de obra durante la cosecha el cerezo se restringió a un $25 \%$, el almendro a un $35 \%$ y el frambueso no más de $20 \%$.

La Tabla 7 muestra el resultado de aplicar estas restricciones en el modelo donde es posible inferir que los cultivos tradicionales como trigo, maíz y alfalfa, no son considerados como una alternativa económicamente viable, excepto en Navidad donde para cuencas tipo "grande" aparece una superficie de 3.15 ha de maíz, lo que demuestra que este cultivo se podría incorporar a la rotación cuando exista una gran disponibilidad de agua.

Tabla 7: Resultados del modelo de optimización y patrón de cultivo por tipo de cuenca, incorporando criterios de diversificación productiva

\begin{tabular}{|l|c|c|c|c|c|c|c|c|}
\cline { 2 - 10 } \multicolumn{1}{c|}{} & \multicolumn{4}{c|}{ Comuna de Navidad } & \multicolumn{3}{c|}{ Comuna de Pumanque } \\
\cline { 2 - 10 } \multicolumn{1}{c|}{} & Unidad & Grande & Med-Gde & Med. & Pequeña & Grande & Med. & Med. \\
\hline Utilidad & USM\$ & 487,170 & 396,086 & 97,739 & 23,138 & 315,204 & 156,975 & 53,009 \\
\hline $\begin{array}{l}\text { Max. } \\
\text { Superficie }\end{array}$ & ha & 47,25 & 39,1 & 11,05 & 3,4 & 32 & 17 & 6,6 \\
\hline $\begin{array}{l}\text { Agua } \\
\text { utilizada }\end{array}$ & Mm $^{3}$ & 323,864 & 241,087 & 63,117 & 19,235 & 182,353 & 107,542 & 37,443 \\
\hline $\begin{array}{l}\text { Req. mano } \\
\text { obra }\end{array}$ & JH & 5945 & 6699 & 1893 & 582 & 5548 & 2913 & 1139 \\
\hline Cerezos & ha & 12,6 & 11,5 & 3,25 & 1 & 9,75 & 5 & 2 \\
\hline Almendro & ha & 22,1 & 16,1 & 4,55 & 1,4 & 13,65 & 7 & 2,8 \\
\hline Frambueso & ha & 6,3 & 9,2 & 2,6 & 0,8 & 7,8 & 4 & 1,6 \\
\hline Alfalfa & ha & 0 & 0 & 0 & 0 & 0 & 0 & 0 \\
\hline Cebolla & ha & 3,15 & 2,3 & 0,65 & 0,2 & 0,82 & 1 & 0,172 \\
\hline Trigo & ha & 0 & 0 & 0 & 0 & 0 & 0 & 0 \\
\hline Maíz & ha & 3,15 & 0 & 0 & 0 & 0 & 0 & 0 \\
\hline
\end{tabular}


En el caso del trigo, no es considerado como alternativa de producción bajo riego en ningún tipo de cuenca, por lo que su cultivo como parte del sistema productivo característico de la zona debería seguir realizándose bajo condiciones de secano.

La alfalfa es un cultivo que permanentemente los agricultores de la zona mencionan como ideal para alimentar su ganado o conservar forraje para épocas de baja disponibilidad, y que se podrían implementar en caso de tener disponibilidad de agua. Los resultados indican claramente que no es una buena alternativa para la condición de secano, debido a su alta demanda hídrica y su bajo precio de venta. Esta situación indica que se deben explorar otras alternativas forrajeras como falaris o mezclas de avena (avena sativa) con vicia (vicia atropurpurea) que se adaptan muy bien a condiciones de secano, dejando así el agua disponible para otros cultivos que produzcan un mayor retorno económico por unidad de agua utilizada.

Respecto del cultivo de cebolla, éste se incluye en todos los patrones de cultivo en superficies desde 0.17 a 3.15 ha. Esto obedece principalmente al nivel de producción que es posible obtener, del orden de $50.000 \mathrm{~kg} / \mathrm{ha}$, lo que compensa el bajo precio que puede presentar el producto. En cuanto a los frutales, éstos forman parte de todos los patrones de cultivo y se manifiestan como la base productiva de cada cuenca analizada. Resalta el comportamiento del almendro, que ocupa en promedio el $42 \%$ de la superficie potencialmente disponible para riego. La razón de esta importante presencia es su alto precio de venta y bajos requerimientos de agua y mano de obra.

La segunda especie frutal en cuanto a superficie ocupada, es el cerezo con un $29 \%$. Esta especie resulta interesante de evaluar en mayor profundidad en la zona de estudio por su característica de producción temprana y concentrada en pocos meses (noviembre y diciembre). Finalmente, la tercera especie frutal en importancia resultó ser el frambueso, que ocupó en promedio el $22 \%$ de la superficie. Su principal limitante es la gran cantidad de mano de obra que se requiere para su cultivo, especialmente en cosecha, lo que repercute en los costos de producción y lo deja como tercera alternativa al momento de decidir la asignación del recurso agua.
Cabe hacer notar que se pueden evaluar muchas más alternativas de cultivos, sin embargo, se considera que los antecedentes expuestos son la base para acciones futuras.

\section{Análisis de sensibilidad}

Villavicencio (2003) realizó un detallado análisis de sensibilidad que permitió evaluar como afectan a los patrones de cultivo las posibles variaciones que puedan tener las variables y parámetros considerados en el modelo de optimización. Estos resultados demuestran que no todas las cuencas son afectadas en la misma proporción ni por los mismos factores. Como tendencia general se advierte que la variable "precio del producto" afecta la función objetivo en forma creciente de mayor a menor tamaño de cuenca, es decir, las de mayor tamaño son menos vulnerables a variaciones de precio que las más pequeñas. Esto se explica por el comportamiento de la función "costo de embalse", lo cual determina que cuencas con menor capacidad de embalse tengan un costo relativo más alto por $\mathrm{m}^{3}$ de agua embalsada que las cuencas más grandes. Por lo tanto, al disminuir la variable "precio del producto", las cuencas pequeñas quedan con costos más altos, a diferencia de las cuencas grandes, donde el costo por $\mathrm{m}^{3}$ de agua embalsada es menor.

La segunda variable de importancia, es la disponibilidad de agua. Su efecto es significativo en todas las cuencas, excepto en las grandes de Navidad, donde el impacto es un $50 \%$ menor que en las demás. En tercer lugar se ubica la disponibilidad de mano de obra en cuencas medianagrandes y pequeñas de Navidad y medianas y pequeñas de Pumanque. Por otra parte la eficiencia del método de riego tiene un efecto creciente desde cuencas mediano-grande a pequeñas en Navidad y en todas las cuencas de Pumanque, sólo en las de tipo grande en Navidad muestra un efecto marginal. Los parámetros que tienen el menor efecto son el costo de mano de obra y el costo de los equipos de riego.

\section{Conclusiones}

La metodología desarrollada facilita el análisis, la toma de decisiones y la focalización de instrumentos y estrategias de planificación del uso de los recursos hídricos en zonas de secano. El uso del modelo de optimización demostró ser una herramienta útil para conciliar el óptimo técnico y 
económico y definir los patrones de cultivo más apropiados dado un conjunto de recursos y restricciones agronómicas, físicas y de mercado.

En orden de importancia, las variables que más influyeron fueron precio de productos, disponibilidad de agua, disponibilidad de mano de obra, costo de mano de obra, eficiencia del método de riego y costo de los equipos de riego. Por otra parte, se determinó una respuesta diferenciada de las cuencas a estas variables, siendo las cuencas pequeñas las más vulnerables a variaciones en estos parámetros. Cabe hacer notar que la metodología aquí desarrollada es factible de replicar en otras zonas donde el recurso agua es escaso y se requiera tener elementos técnicos de decisión al momento de definir estrategias de intervención en dichas zonas. Lo anterior entrega valiosos elementos de análisis que permiten con la ilustración de escenarios prever posibles impactos de políticas o medidas de fomento tendientes a fortalecer la producción agrícola en zonas con restricción hídrica

Es importante considerar que la intervención en estas zonas no debe entenderse como una transformación del secano en una zona de riego, sino que, a partir de sus limitantes y potencialidades, usar racionalmente el agua factible de acumular durante el invierno y conjugarlo con la incorporación de especies que se adapten a condiciones de restricción hídrica, de modo de configurar un sistema productivo más estable, diversificado y capaz de sustentar a la población rural que habite en él.

\section{Referencias}

Al-Jamal, M.S., Sammis, T.W., Ball, S. and Smeal, D. (2000). Computing the crop water production function for onion. Agricultural Water Management 46, No. 1, 29-41

Billib, M., Holzapfel, E.A. and Fernandez-Cirelli, A. (2009). Sustainable water resources management for irrigated agriculture in Latin America. Chilean Journal of Agricultural Research 69, suppl.1, 3-5

Brooke, A., Kendrick, D. and Meeraus, A. (1988). GAMS $A$ user's guide. The Scientific Press. Redwood City, U.S.A.

Carvallo, H.O, Holzapfel, E.A., Mariño, M.A. and López, M.A.
(1988). Irrigated cropping optimization. Journal of Irrigation and Drainage Engineering 124, No. 2, 67-72

CIREN -CORFO (1996). Manual de obras menores de riego. Publicación Ciren n ${ }^{\circ}$ 111. Santiago, Chile

Torrecillas, A., Ruiz -Sanchez, M.C., Leon, A. and Del Amor, F. (1989). The response of young almond trees to different drip irrigated conditions, development and yield. Journal of Horticultural Science and Biotechnology 64, No.1, 1-7

Uribe, H., Arumí, J.L., González, L. and Salgado, L.G. (2003). Groundwater recharges using hydrological balances in the central drylands of Chile. Ingeniería Hidráulica en México 18, No. $3,17-28$

Villavicencio, A. (2003). Identificación y planificación del uso de recursos hídricos en zonas de secano. Tesis de Magister en Ingeniería Agrícola, Universidad de Concepción, Chile 